\title{
Preface: Special Session SpS16 Unexplained Spectral Phenomena in the Interstellar Medium
}

There are several outstanding mysteries in interstellar medium spectroscopy which have remained unsolved after decades of effort. The diffuse interstellar bands (DIBs) have been known for almost a century (Heger 1922). Although more than 400 bands from the near UV to near infrared have been detected, none of them has been identified. In the Milky Way Galaxy, DIBs have been seen towards over one hundred stars. In the Magellanic Clouds, DIBs have been seen in the spectrum of SN 1987A as well as in the spectra of reddened stars (Ehrenfreund et al. 2002). DIB carriers in the interstellar medium of external galaxies can be probed by supernovae (Sollerman et al. 2005), and DIBs have been detected in external galaxies with redshifts up to 0.5 (Sarre 2006).

The $217.5 \mathrm{~nm}$ extinction feature has been known for about 45 years (Stecher 1965). It was extensively observed by the International Ultraviolet Explorer (IUE) satellite and is found to have remarkable constancy in its peak wavelength of $217.5 \mathrm{~nm}$, corresponding to $5.7 \mathrm{eV}$. This is not just a local phenomenon as the feature has been detected in galaxies as distant as redshift $\mathrm{z}>2$ (Elíasdóttir et al. 2009).

A family of unidentified infrared emission (UIE) features was discovered over 30 years ago (Russell et al. 1977) and the number of features has been expanding as the result of infrared spectroscopic observations from ISO and Spitzer. The UIE phenomenon include aromatic bands at $3.3,6.2,7.7,8.6$, and $11.3 \mu \mathrm{m}$, aliphatic features at 3.4 and $6.9 \mu \mathrm{m}$, broad emission plateaus at 8,12 , and $17 \mu \mathrm{m}$, as well as a host of weaker features that are too broad to be atomic or molecular lines. The UIE features are seen in very different radiation environments. The energy source responsible for the excitation of the features ranges from tens of thousands of degrees in planetary nebulae, $\sim 30000 \mathrm{~K}$ in HiI regions, to thousands of degrees in reflection nebulae and proto-planetary nebulae. Although the UIE phenomena have been widely suggested to be due to polycyclic aromatic hydrocarbon (PAH) molecules, other forms of carbonaceous materials have also been under discussion.

The observation of the Extended Red Emission (ERE) also goes back 30 years. ERE is commonly seen in reflection nebulae (Witt \& Schild 1988, Witt \& Boroson 1990). It has also been detected in dark nebulae, cirrus clouds, planetary nebulae, HiI regions, the diffuse interstellar medium, and in haloes of galaxies. The central wavelength of the emission shifts from object to object, or even between locations within the same object. Other than the fact that it may be due to photoluminescence, exact nature of its carrier is still unknown. Other unidentified optical emissions include a set of bright visible bands seen alongside ERE in the Red Rectangle nebula (Schmidt \& Witt 1991) and Blue Luminescence which is seen both in this object and a number of other sources (Vijh, Witt \& Gordon 2004).

The 21 and $30 \mu \mathrm{m}$ unidentified infrared features are generally associated with objects in the late stages of stellar evolution (Kwok et al. 1989, Forrest et al. 1981), and can be responsible for a significant fraction of the energy output of the stellar sources (Hrivnak et al. 2000). High resolution $I S O$ observations have found that the $21 \mu \mathrm{m}$ features have the same intrinsic profile and peak wavelength $(20.1 \mu \mathrm{m})$ (Volk et al. 1999). There is no evidence for any discrete sub-structure due to molecular bands in the observed spectra, 
suggesting that the $21-\mu \mathrm{m}$ feature is either due to a solid substance or a mixture of many similarly structured large molecules.

It is interesting to note that these phenomena have been observed not only in the diffuse ISM, but also in circumstellar environments, in the galactic halo, and in external galaxies. In some cases they have been observed in galaxies with high redshifts, suggesting that the carriers responsible for these features were already present in the early Universe.

We now recognize that all these UV, optical and infrared spectral features are ubiquitous in the Universe implying that their carriers must be a substance made of common elements, most likely carbon. The detection of over 170 different molecular species (many of them organic) in the millimeter-wave and infrared spectral regions through their rotational and vibrational transitions. Complex organics are also found in meteorites, interplanetary dust particles, and planetary surfaces. These new developments have shown that the Universe is filled with organics (Kwok 2011). There is a possibility that the carriers of are of organic composition but the relevant materials are not naturally present in the terrestrial environment. The recent detections of $\mathrm{C}_{60}$ and $\mathrm{C}_{70}$ in planetary nebulae (Cami et al. 2010, Garćia-Hernández et al. 2010, Zhang \& Kwok 2011) and reflection nebulae (Sellgren et al. 2010) have also raised interest in other carbon allotropes in the ISM.

The Special Session 16 was held on August 27-28 at the IAU GA in Beijing. We were able to bring together observers who reported on the latest measurements of these features, modelers who use molecular data to interpret the observations, and laboratory spectroscopists who provide expertise in helping the identification. Some significant new results were reported. We are hopeful that this Special Session will be remembered as a major step towards the solution to these long standing mysteries.

\section{References}

Cami, J., Bernard-Salas, J., Peeters, E., \& Malek, S. E. 2010, Science 329, 1180

Ehrenfreund, P. et al. 2002, ApJ 576, L117

Elíasdóttir, Á. et al. 2009, ApJ 697, 1725

Forrest, W. J., Houck, J. R., \& McCarthy, J. F. 1981, ApJ 248, 195

Garćia-Hernández, D. A. et al. 2010, ApJ 724, L39

Heger, M. L. 1922, Lick Observatory Bulletin 337, 141

Hrivnak, B. J., Volk, K., \& Kwok, S. 2000, ApJ 535, 275

Kwok, S. 2011, Organic Matter in the Universe, Wiley

Kwok, S., Volk, K. M., \& Hrivnak, B. J. 1989, ApJ 345, L51

Russell, R. W., Soifer, B. T., \& Willner, S. P. 1977, ApJ 217, L149

Sarre, P. J. 2006, J. Mol. Spectrosc. 238, 1

Schmidt, G. D., \& Witt, A. N., 1991, ApJ 383, 698

Sellgren, K. et al. 2010, ApJ 722, L54

Sollerman, J. et al. 2005, A\&A 429, 559

Stecher, T. P. 1965, ApJ 142, 1683

Vijh, U. P. et al. 2004, ApJ 606, L65

Volk, K., Kwok, S., \& Hrivnak, B. J. 1999, ApJ 516, L99

Witt, A. N. \& Boroson, T. A. 1990, ApJ 355, 182

Witt, A. N. \& Schild, R. E. 1988, ApJ 325, 837

Zhang, Y. \& Kwok, S., 2011, ApJ 730, 126.

I want to thank Anisia Tang for her assistance in organizing this Special Session and the Local Organizing Committee on their help in the logistical arrangements.

Sun Kwok, Chair SOC,

Hong Kong, China, November 30, 2012 